Original paper

\title{
Dose calculation deviations induced by fractional image-guided-couch-shifts for Varian Halcyon MV cone beam CT
}

\author{
Yuliang Huang ${ }^{\mathrm{a}, 1}$, Hao $\mathrm{Wu}^{\mathrm{a}, 1}$, Qiaoqiao $\mathrm{Hu}^{\mathrm{a}}$, Meijiao Wang ${ }^{\mathrm{a}}$, Ruoxi Wang ${ }^{\mathrm{a}}$, Zhixin $\mathrm{Zu}^{\mathrm{b}}$, \\ Haiyang Wang ${ }^{\mathrm{a}}$, Weihu Wang ${ }^{\mathrm{a}}$, Yibao Zhang, ${ }^{\mathrm{a}, *}$ \\ ${ }^{a}$ Key Laboratory of Carcinogenesis and Translational Research (Ministry of Education/Beijing), Department of Radiation Oncology, Peking University Cancer Hospital \& \\ Institute, Beijing 100142, China \\ ${ }^{\mathrm{b}}$ Varian Medical Systems, 8 Yuncheng Street, BDA Beijing 100176, China
}

\section{A R T I C L E I N F O}

\section{Keywords:}

Halcyon

MVCBCT

Imaging dose

IGRT

\begin{abstract}
A B S T R A C T
Purpose: To assess the criticality of calculation deviations induced by fractional image-guided-couch-shifts for Halcyon MV cone beam CT (CBCT) dose, which is incorporated as part of total treatment dose.

Methods: Eclipse-calculated imaging dose was first validated in 'Cheese Phantom' by measurement. Then, the actual imaging dose $\left(\mathrm{D}_{\text {act }}\right)$ for 18 historical patients of various sites were recalculated based on 513 MV CBCTguided-couch-shift data, and compared with reference computations based on treatment isocentre ( $\left.D_{\text {ref }}\right)$. Patientand plan-specific dose from treatment fields was integrated with $\mathrm{D}_{\text {act }}$ and $\mathrm{D}_{\text {ref }}$ respectively for comparison. Results: The average absolute relative disagreements between the measured and calculated dose were less than $1.23 \%$. The mean $\pm 1 S D$ of gamma passing rates of the accumulated imaging dose and total dose were $80.71 \pm 6.22 \%$ and $99.81 \pm 0.32 \%$ respectively based on $3 \mathrm{~mm} / 3 \% /$ local $/ 10 \%$ threshold criteria. The accumulated errors of minimum imaging dose to PTV were no larger than $-14.38 \mathrm{cGy}$, which were reduced to $-0.82 \mathrm{cGy}$ after the heterogeneous treatment dose was overlaid. The mean relative discrepancies of PTV minimum dose were $-0.61 \mathrm{cGy}(-0.71 \%)$ and $-0.00(0.00 \%)$, before and after incorporating the treatment dose respectively.

Conclusions: The Eclipse-calculated Halcyon MV CBCT dose was validated. Although the isocentre displacementinduced imaging dose calculation errors for Halcyon MV CBCT were partially cancelled out by couch shifts of various directions and distances, especially after the incorporation of heterogeneous treatment dose, it was still advisable to monitor the accumulated deviations and replan when unacceptable target under-dose or organ overdose were observed.
\end{abstract}

\section{Introduction}

As the most commonly used modality for image-guided radiotherapy (IGRT), tremendous efforts have been made to measure [1-3], compute [4-5], and reduce [6-7] the excessive imaging dose from $\mathrm{kV}$ cone beam CT (CBCT), which cannot be computed accurately nor be incorporated to the accumulated patient dose by the commercial treatment planning systems (TPS) due to the absence of $\mathrm{kV}$ beam models [8].

To improve the positioning accuracy, pre-treatment MV imaging becomes a mandatory process for Varian Halcyon system V.1.0 (kV imaging is available on V.2.0), which may accumulate more imaging dose from the daily repetition and higher fractional dose of MV CBCT than that of kV CBCT [9-10]. However, using the same beams as for the treatment, Halcyon MV CBCT dose can be automatically calculated by the Eclipse TPS (V.15.1 and later) and incorporated with the treatment dose for a collective dosimetric evaluation. However, it was noticed that the computation was based on the treatment isocentre, from which the actual imaging centre may deviate from one fraction to another, as represented by the couch shift to realign the patient from the initial setup position (imaging centre) to the anatomy-registered treatment isocentre.

Probably due to limited patients treated on this very new Halcyon system worldwide, it has not been reported but clinically desirable to appreciate whether this systematic deviation can be amplified by dozens of imaging repetitions, or to the contrary, potentially smeared

\footnotetext{
* Corresponding author at: Department of Radiation Oncology, Beijing Cancer Hospital, 52 Fucheng Road, Haidian, Beijing 100142, China.

E-mail address: zhangyibao@pku.edu.cn (Y. Zhang).

${ }^{1}$ Yuliang Huang and Hao Wu contributed equally to this work.
} 
by various distances and directions of the isocentre shift bringing the dose up or down.

Based on 513 clinical MV CBCT scans of various anatomic sites, the associated couch shift data of 18 patients that were treated on Halcyon were used to retrospectively calculate the accumulative imaging dose discrepancies induced by the isocentre shift, aiming to evaluate its criticality and assist clinicians to make a re-planning decision if severe target under-dose or OAR over-dose were observed, by monitoring the accumulated deviations throughout the treatment course.

\section{Material and methods}

This retrospective, computation-based study has been approved by the Institutional Medical Ethics Committee with a waiver of informed consent.

\subsection{Varian Halcyon and the MV CBCT system}

As Varian's newest cancer treatment device, Halcyon has a lot of new designs compared with the conventional machines, such as: a closed ring gantry allowing a maximum rotation speed of 4 RPM between $\pm 185^{\circ}$; a beam stopper in the primary beam path behind the portal imager to minimize the room shielding requirement; on-board laser systems and touch screens; elimination of the need for steering coils, bending magnet, jaws, field light, beam line accessories, optical distance indicator, and user pendants, etc. Both imaging and treatment use identical 6-MV flattening-filter-free photon beams, as collimated by a dual-layer multi-leaf collimator (MLC) system of $1.0 \mathrm{~cm}$ leaf width to the isocentre plane shaping a maximum field size of $28.0 \mathrm{~cm} \times 28.0 \mathrm{~cm}$. An offset of $0.5 \mathrm{~cm}$ between the proximal and distal MLC stacks, combined with taller leaves and larger diameter of the rounded leaf ends, have significantly reduced the penumbra, leaf transmission and leakage relative to the conventional MLCs. Other new features include doubled leaf speed (than Millennium 120 MLC), 100\% of leaf over-travel and inter-digitations, etc.

The MV CBCT uses an imaging panel of $43.0 \mathrm{~cm} \times 43.0 \mathrm{~cm}$ containing $1280 \times 1280$ pixels, $154.0 \mathrm{~cm}$ away from the source. Two dose rates, $45 \mathrm{MU} / \mathrm{min}$ and $27 \mathrm{MU} / \mathrm{min}$ are used to acquire 'High-Quality' and 'Low-Dose' MV CBCT images respectively: both scans rotate the gantry from $260^{\circ}$ to $100^{\circ}$, clockwise. The 'High Quality' and 'Low Dose' mode delivers $10 \mathrm{MU}$ and $5 \mathrm{MU}$ per scan respectively. The maximum reconstructed imaging diameter and length are $27.7 \mathrm{~cm}$ and $26.0 \mathrm{~cm}$ respectively, using a radiation field of $28.0 \mathrm{~cm} \times 28.0 \mathrm{~cm}$ (isocentre plane). Only the imaging length (patient superior-inferior direction) is user-definable within the physical dimension limit of $28 \mathrm{~cm}$, because the DMI (Digital Megavoltage Imager) is fixed to the beam stopper and is always in the beam path, which cannot be shifted to enlarge the fieldof-view, known as half-fan scanning on the conventional $\mathrm{kV}$ CBCT systems.

\subsection{Measurement based validation of imaging dose computation}

To validate the imaging dose computed on Eclipse, a calibrated A1SL chamber with temperature and air pressure corrections (Standard Imaging, Middleton, WI) was used to measure the Halcyon MV CBCT dose (scanning length: $28 \mathrm{~cm}$; High Quality mode) to 28 cavities along a linear array in a homogeneous 'Cheese Phantom', which is a standard imaging dose and quality verification device for the TomoTherapy MV CT (Accuray, Sunnyvale, CA). Although Halcyon uses MV CBCT and its treatment techniques are quite different from that of TomoTherapy, 'Cheese Phantom' has been one of the most commercially available QA devices for MV imaging so far, before a dedicated tool for Halcyon is developed. The diameter and thickness of the cheese phantom are $30.0 \mathrm{~cm}$ and $18.0 \mathrm{~cm}$ respectively. Considering the asymmetric scanning beam angles of Halcyon MV CBCT, the linear array was measured on both horizontal (Fig. 1-a) and vertical (by rotating the phantom by

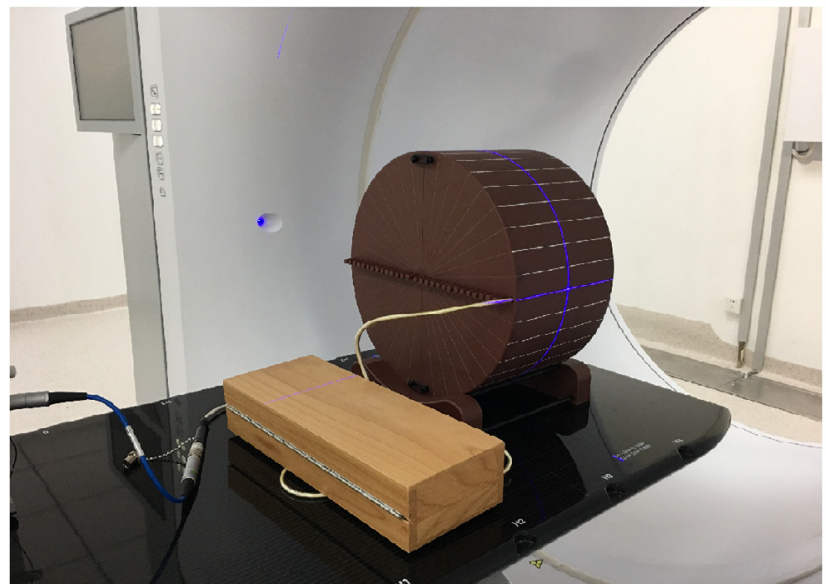

(a)
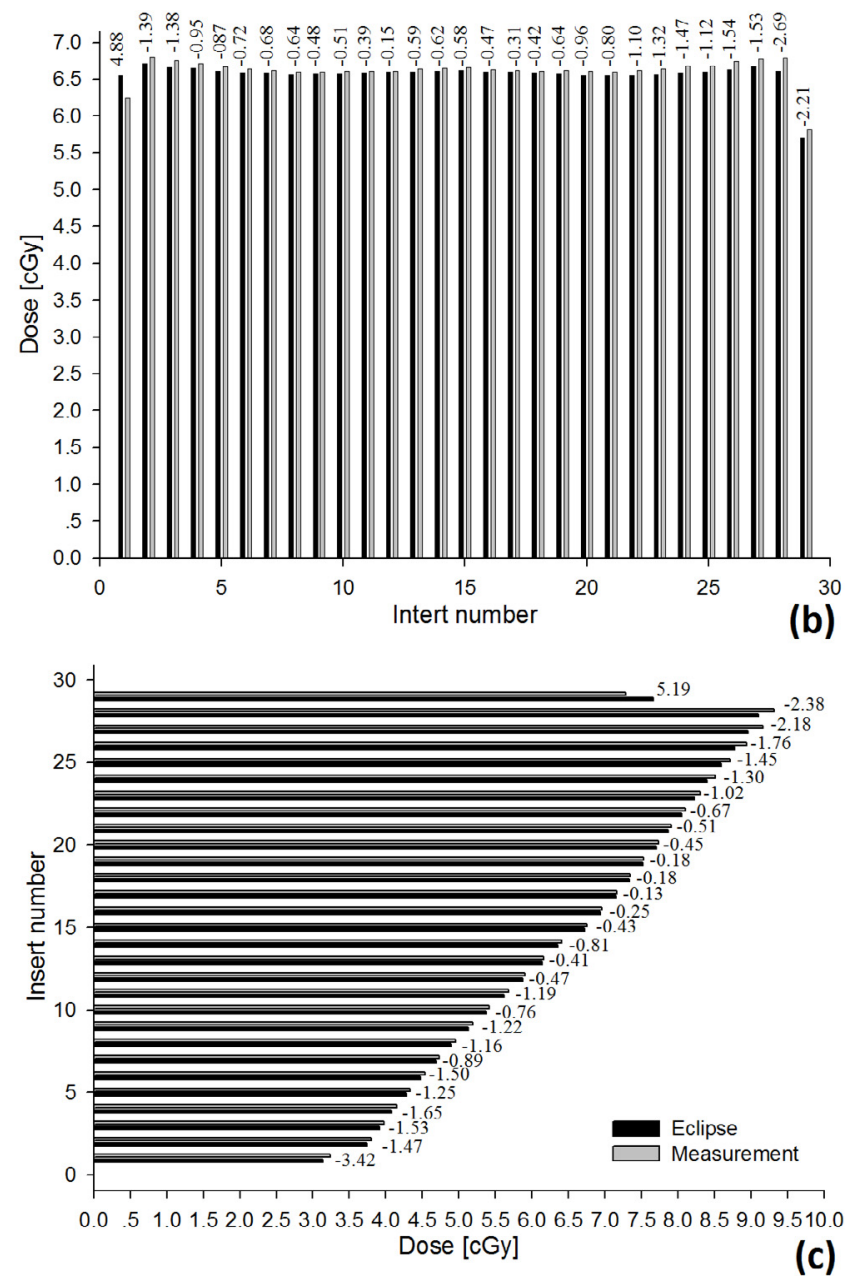

Fig. 1. (a) Horizontal setup of Cheese phantom; (b/c) Horizontal/vertical dose deviations in the cheese phantom between the Eclipse calculations (black) and chamber measurements (gray). The numerical deviations [\%] labelled beside the bars were calculated as: (Eclipse-Measurement)/Measurement*100.

$90^{\circ}$ from the setup of Fig. 1-a) directions. All unmeasured cavities were filled with the water-equivalent inserts. As comparison, the imaging dose to each corresponding measurement point was calculated by Eclipse on the planning CT data sets of the 'Cheese Phantom', using the same imaging centre. 
Table 1

The lengths [cm] of PTV and MV CBCT imaging fields, and the scanning mode of each patient*fractions.

\begin{tabular}{llllllll}
\hline \multirow{2}{*}{ ID } & \multirow{2}{*}{ Length } & \multicolumn{2}{c}{ Mode } & ID & \multicolumn{2}{c}{ Length } & \multirow{2}{*}{ Mode } \\
\cline { 2 - 3 } \cline { 6 - 7 } & PTV & CBCT & & & PTV & CBCT & \\
\hline HN1 & 14.1 & 18.0 & HQ*15 & Thx1 & 16.0 & 14.0 & HQ*31 \\
HN2 & 20.4 & 14.0 & HQ*34 & Thx2 & 11.5 & 14.0 & HQ*35 \\
HN3 & 18.9 & 18.0 & HQ*33 & Thx3 & 16.5 & 14.0 & HQ*28 \\
Abd1 & 6.0 & 20.0 & HQ*34 & Thx4 & 11.0 & 20.0 & HQ*25 \\
Abd2 & 12.5 & 14.0 & HQ*25 & Thx5 & 9.5 & 20.0 & HQ*26 \\
Abd3 & 18.0 & 14.0 & HQ*33 & Thx6 & 21.0 & 14.0 & LD*23 \\
Pel1 & 19.5 & 14.0 & LD*31 & Thx7 & 9.5 & 14.0 & LD*22 \\
Pel2 & 16.5 & 20.0 & HQ*33 & Thx8 & 12.0 & 10.0 & LD*25 \\
Pel3 & 24.0 & 20.0 & HQ*30 & Leg1 & 22.0 & 20.0 & LD*30 \\
\hline
\end{tabular}

Abbreviations: $\quad \mathrm{HN}=$ head \& neck; $\mathrm{Abd}=$ Abdominal; $\mathrm{Thx}=$ Thoracic; Pel = Pelvic; HQ = High Quality; LD = Low Dose.

\subsection{Imaging dose deviations induced by couch shifts}

Three patients were used to display the deviations of dose distributions explicitly on the CT anatomies of head, thorax and pelvis respectively. As an example, a $5.0 \mathrm{~mm}$ shift on the vertical, longitudinal and lateral directions were introduced to the treatment isocentres simultaneously, mimicking the actual imaging isocenter. Using Eclipse AAA_15017 algorithm, the imaging dose calculated based on the treatment isocentre (reference) and imaging isocentre (actual) were denoted as $D_{\text {ref }}$ and $D_{\text {act }}$ respectively. The $D_{\text {act }}$ distributions of a single
'High Quality' scan were subtracted from the corresponding $D_{\text {ref }}$ and the results were plotted using Varian Velocity software (V.3.2.1). The results of 'Low Dose' scan can be approximated as half of that of 'High Quality' mode [11].

To numerically investigate the accumulative effect of repetitive scanning based on real clinical data, 513 MV CBCT of various disease sites and their corresponding couch shifts of 18 historical patients that were treated on Halcyon were retrospectively selected. Table 1 shows the lengths of PTV and the MV CBCT imaging fields defined as length alongside the longitudinal direction. With limited experience on Halcyon, the imaging length and scanning mode selected for each patient were at the clinician's discretion. The treatment isocentre was coincident with the centre of planning target volume (PTV), based on which accumulated $\mathrm{D}_{\text {ref }}$ was computed using the patient specific imaging mode, field size and fractions. For efficiency, an Eclipse Scripting Application Programming Interface (ESAPI) script was created to automate the recalculations of $\mathrm{D}_{\text {act }}$ by including the displaced isocentres based on fractional couch shifts. The imaging dose agreement between the $\mathrm{D}_{\text {ref }}$ and $\mathrm{D}_{\text {act }}$ accumulated throughout the whole course was compared using Gamma analysis, based on the criteria of $3 \mathrm{~mm} / 3 \% / \mathrm{local} /$ $10 \%$ threshold. In addition, the Eclipse-calculated patient- and planspecific dose from the treatment fields was integrated with $D_{\text {ref }}$ and $D_{\text {act }}$ imaging dose respectively, yielding 'total dose' for comparison. In addition to the holistic Gamma analysis, the target cold spots and mean dose were also compared specifically to investigate the criticality of the imaging dose uncertainties.

This work used Gamma passing rate for an overall disparity assessment, and focused specifically on the target dose uncertainties
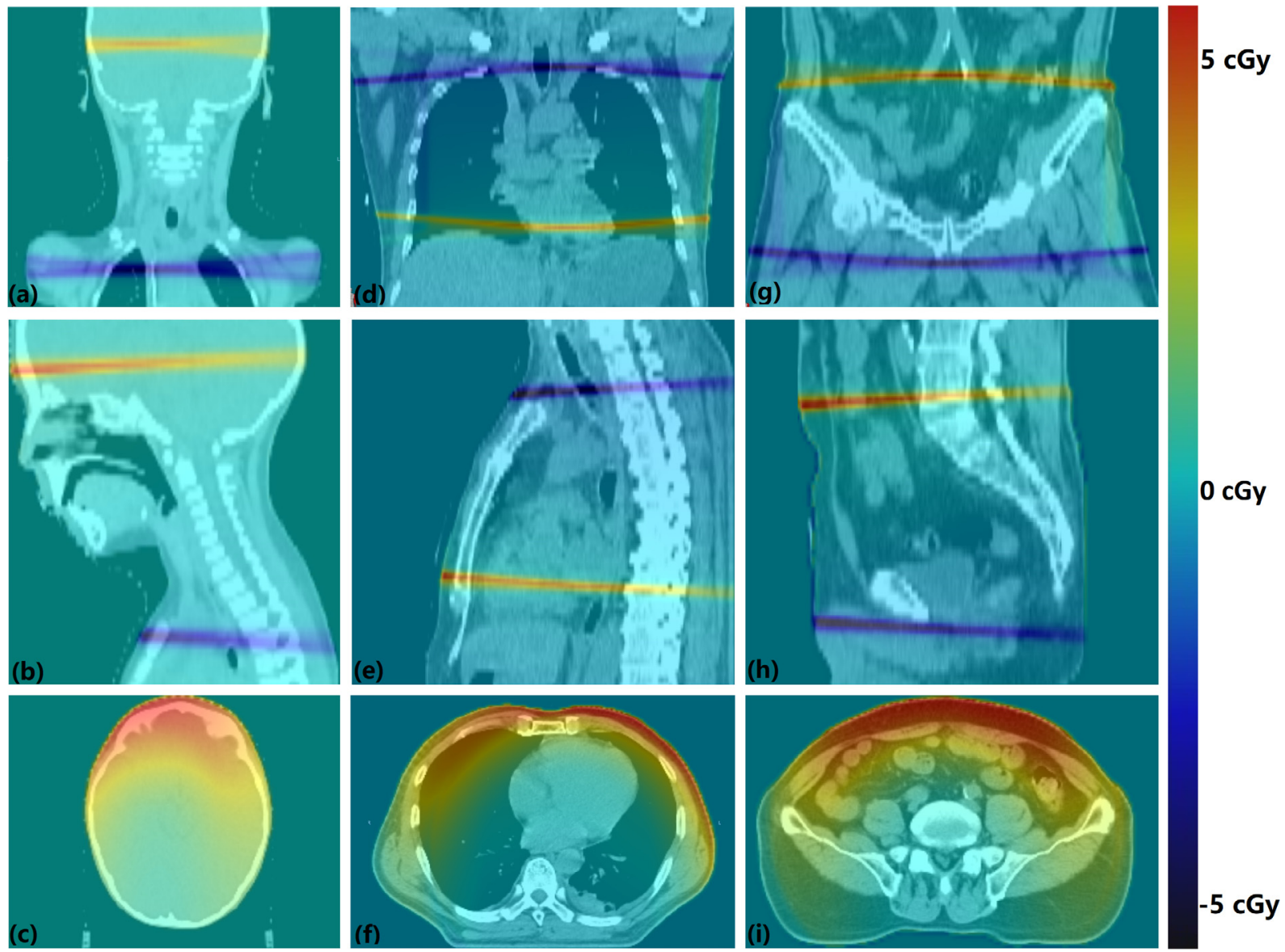

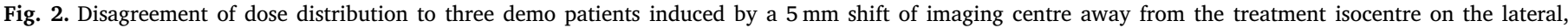

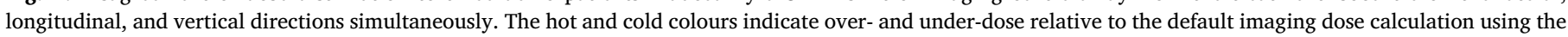

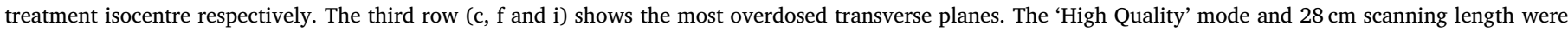
used as examples. 
because: 1. All patients with various diseases have this structure in common, which also determines the treatment isocentre at our centre; 2. The individual OAR dose is strongly dependent on patient-specific anatomy, scanning protocol, fractional imaging isocentre and user-defined imaging length. Absolute OAR dose can be found in a more transferable standardized phantom study [11].

\section{Results}

\subsection{Measurement based imaging dose validation}

Fig. 1 displays the dose to the cavity arrays in the Cheese Phantom, where subfigures (a-b) indicate the horizontal setup and the corresponding dose results respectively. Subfigure (c) shows the results of the vertical setup by rotating the phantom by $90^{\circ}$. The numbers between the bars show the disagreements [\%] between the Eclipse calculations (black) and chamber measurements (gray), calculated as (Eclipse-Measurement)/Measurement*100, where the signed and absolute mean $\pm 1 \mathrm{SD}$ were $-0.75 \% \pm 1.22 \%$ and $1.08 \% \pm 0.93 \%$ for horizontal, $-0.88 \% \pm 1.38 \%$ and $1.23 \% \pm 1.06 \%$ for vertical respectively.

\subsection{Dose error induced by isocentre shift}

Fig. 2 displays examples of the dose distribution disparities between $D_{\text {ref }}$ and $D_{\text {act }}$ on the CT images of a head and neck (a, b, c), a thoracic ( $d$, e, f) and a pelvic patient ( $g, h, i)$, as induced by a $5.0 \mathrm{~mm}$ shift on the lateral, vertical and longitudinal directions simultaneously. The hot and cold colours on the heat maps indicate the over- and under-dose relative to $\mathrm{D}_{\text {ref }}$ respectively. The volumes between the hottest and coldest regions were within the imaging fields of $28.0 \mathrm{~cm}$. The subfigures (c, f, i) show the transverse sections that were most over-dosed.

The imaging dose recalculation based on the fractional couch shift was successfully automated by the ESAPI script, which took less than 30 s per patient based on a standalone workstation (Intel Xeon CPU E78860 v4 @2.20 GHz, 4 processors, 128 GB RAM). Fig. 3 displays the 513 couch shifts on the lateral (a), vertical (b) and longitudinal (c) directions of 18 patients that were treated on Halcyon and guided by MV CBCT fractionally.

Based on the couch shifts shown in Fig. 3, Table 2 displays the deviations between $D_{\text {ref }}$ and $D_{\text {act }}$ in the PTV accumulated throughout the whole treatment course, before and after the treatment dose was incorporated. The negative values indicate the $\mathrm{D}_{\text {act }}$ was lower than the $\mathrm{D}_{\text {ref. }}$

\section{Discussion}

As shown in Fig. 1, the maximum dose deviations between the Eclipse calculations (black) and chamber measurements (gray) were all observed at the superficial area. The far left (4.88\%), right $(-2.21 \%)$, upper $(5.19 \%)$ and lower $(-3.42 \%)$ cavities were all within a distance of $1.5 \mathrm{~mm}$ or $6.5 \mathrm{~mm}$ to the surface, where the lack of charged particle equilibrium can be challenging for both chamber measurement and TPS calculation [12]. Without those four spots, the Eclipse calculations were slightly but consistently lower than the chamber measurements. Multiple factors may have contributed jointly to the discrepancies: 1 . It was technically difficult to accurately delineate the 0.053 cc collecting volume of A1SL chamber on Eclipse; 2. Although the delineated volumes were converted to high resolution segments before calculation, the grid size sampling was still limited considering the small collecting volume; 3. The nominal calibration uncertainty of the chamber was $1.40 \%$, which might be larger when detecting very low dose such as imaging exposure; 4 . The machine output deviations within $2-3 \%$ were tolerated clinically, which may differ from the TPS calculation in a single direction when the measurement was performed consecutively in a short period of time. The observed magnitudes of disparities were comparable to the anthropomorphic phantom studies [11]. Considering the maximum disparities $(-2.69 \%$ for horizontal and $-2.38 \%$ for vertical respectively) of non-superficial spots were within the clinical tolerance of $3.00 \%$, Eclipse-computed imaging dose was considered as acceptable for the purpose of this study.

Fig. 1 also displays more heterogeneous dose distribution vertically (c) than horizontally (b), as a result of upper partial arc scanning. Similar heterogeneity was observed on the patient anatomies in the Fig. 3 (c, f, i). Except the most superior point (\#28) in the build-up region as shown in Fig. 1, the dose decreased monotonically downwards.

As shown in Fig. 2, the dose deviations of a single scan were more pronounced at the longitudinal imaging field borders: over-dose to one end but under-dose to the other end (in-field $v s$. out-of-field volumes). Although the beams were un-flattened, the impact of lateral and vertical couch shifts, and the dose deviations of the 'always-in-field volumes' were relatively less prominent than that of the longitudinal edges under the same plotting scale.

Although the maximum imaging dose disparities of a single scan were of the magnitude of $\pm 5 \mathrm{cGy}$ as shown in Fig. 2, the errors were not multiplied by repetitions. Instead, the accumulations were largely neutralized by dozens of couch shifts of 'random' directions and distances. As shown in Table 2, the Gamma passing rates were $80.71 \pm 6.22 \%$ and $99.81 \pm 0.32 \%$ before and after incorporating the treatment dose respectively, which were primarily contributed by the 'always-in-field volumes' as shown in Fig. 2. The failed regions were largely located at the field edges of high dose gradient, which is less deductable by the imaging dose alone. The Gamma passing rates of imaging dose were based on relative dose hence independent from the scanning mode, providing the dose of 'High Quality' and 'Low Dose' were almost proportional [11].

Table 2 also suggests that the accumulated imaging cold spots can be well compensated by superposition with heterogeneous treatment dose distributions: The cold spot of imaging dose $\left(\mathrm{PTV}_{\min }\right)$ was $-14.38 \mathrm{cGy}$ or $-14.23 \%$ lower than that was planned for HN3 patient (the largest magnitude observed in Table 2), which was reduced to $-0.82 \mathrm{cGy}$ or $-0.02 \%$ after the incorporation of treatment dose (PTV $_{\text {min }}$ of the total dose). The maximum deficiency of imaging-contributed PTV mean dose was $-0.79 \mathrm{cGy}$ (Thx3), yet accounted for $-0.01 \%$ only in the total PTV mean dose. For all 18 patients in Table 2, the total dose errors were negligible and all within $-2.26 \mathrm{cGy} \sim 2.33 \mathrm{cGy}$, or $-0.04 \% \sim 0.03 \%$.

This retrospective work is limited by the small patient volume that had been recruited into a terminated Halcyon clinical trial, hence the couch shifts and associated dose deviations were very preliminary. Although the consistent overlapping chance of fractional cold spots or hot spots is small, it is not impossible in theory. Considering the isocentre inconsistency is a systematic, unnecessary and avoidable uncertainty, it is recommendable to apply the daily couch shifts to the imaging dose calculation and monitor the accumulated imaging dose deviation throughout the treatment course. Possible re-planning order is at clinician's discretion if severe target under-dose or OAR over-dose are observed.

\section{Conclusions}

This work validated the Eclipse-calculated Halcyon MV CBCT dose, and demonstrated that the isocentre displacement from MV CBCT to treatment can induce deviations of imaging dose calculations. Although the uncertainties can be partially smeared by couch shifts of various directions and distances, especially after the incorporation of heterogeneous treatment dose, it is still advisable to monitor the accumulated deviations using the fractional couch shifts.

\section{Competing interests}

This work was partially supported by Varian Funding. Yibao Zhang, 

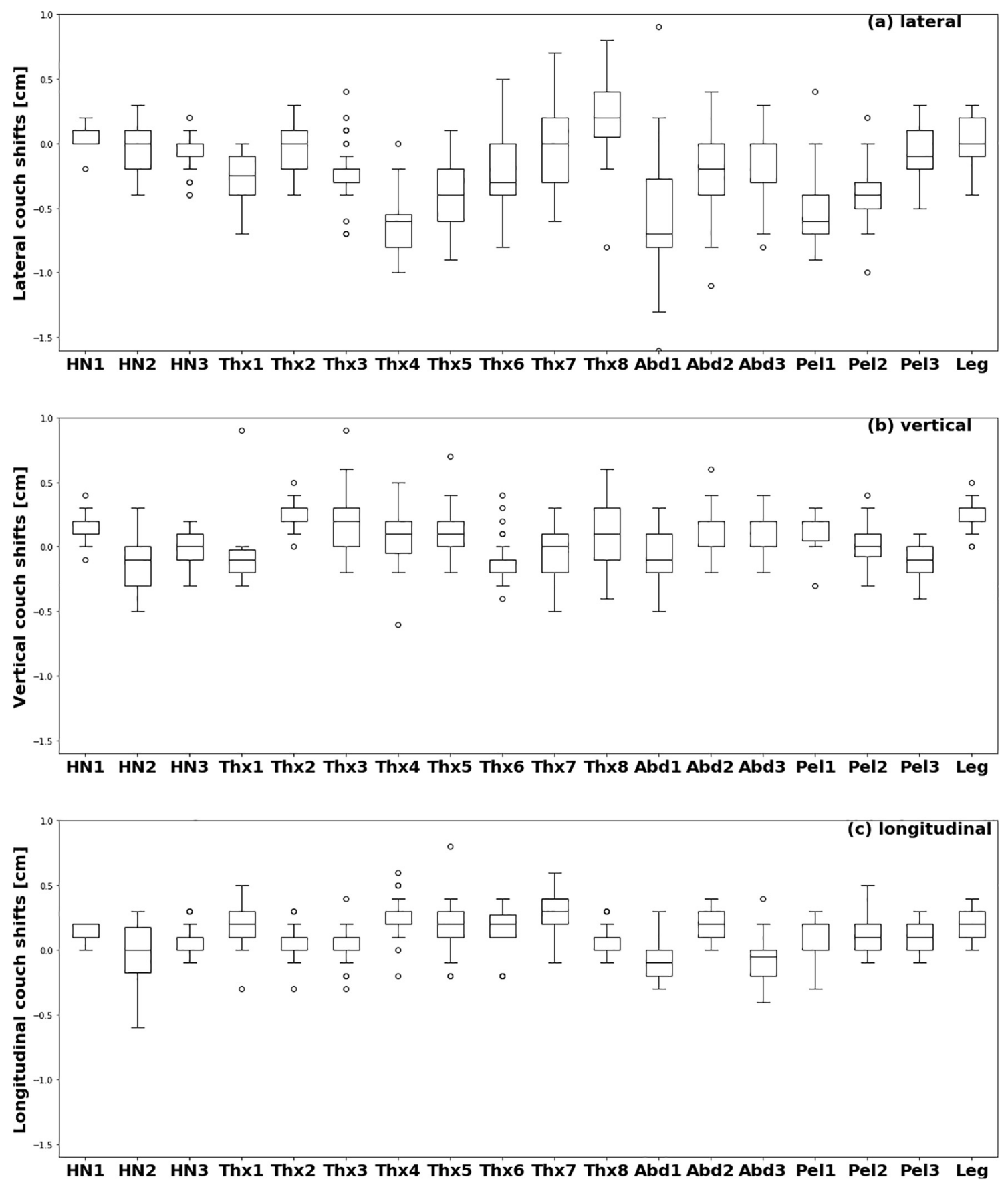

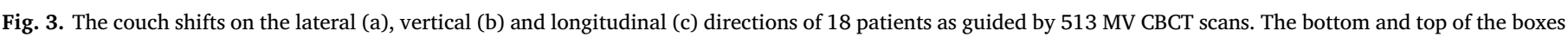

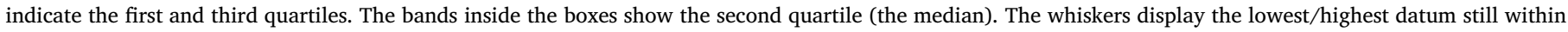
1.5 interquartile ranges of the lower/upper quartiles. The circles indicate the outliers.

Hao Wu and Weihu Wang received speaker's honorarium from Varian Medical Systems. Zhixin Zu is an employee of Varian Medical Systems. This work was jointly supported by Capital's Funds for Health Improvement and Research (2018-4-1027), National Natural Science Foundation of China (11505012), Beijing Natural Science Foundation (7172048 and 1184014), Fund for Fostering Young Scholars of Peking University Health Science Centre (BMU2017PY028), Foundation of Science and Technology Department of Sichuan Province (2018HH0099), Beijing Municipal Administration of Hospitals Incubating Program (PX2019042 and PX2016060) and Beijing Municipal Administration of Hospitals Youth Program (QML20171104).

\section{Acknowledgements}

This work was jointly supported by Capital's Funds for Health Improvement and Research (2018-4-1027), National Natural Science Foundation of China (11505012), Beijing Natural Science Foundation (7172048 and 1184014), Fund for Fostering Young Scholars of Peking University Health Science Centre (BMU2017PY028), Foundation of Science and Technology Department of Sichuan Province (2018HH0099), Beijing Municipal Administration of Hospitals Incubating Program (PX2019042 and PX2016060) and Beijing Municipal Administration of Hospitals Youth Program (QML20171104). The authors thank Mu Yong Lee, Lisa Levine, Jian Zhao, Fei Xia, Shengqiang Leng and Zhihong Pei for their assistance. 
Table 2

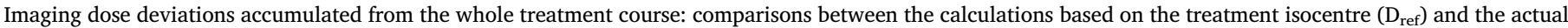

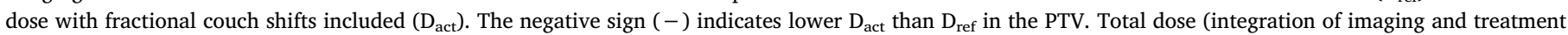
dose) were also compared.

\begin{tabular}{|c|c|c|c|c|c|c|}
\hline & \multicolumn{3}{|c|}{ Imaging Dose Deviation } & \multicolumn{3}{|c|}{ Total Dose Deviation } \\
\hline & Gamma [\%] & $\mathrm{PTV}_{\min } \mathrm{cGy}(\%)$ & $\mathrm{PTV}_{\text {mean }} \mathrm{cGy}(\%)$ & Gamma [\%] & $\mathrm{PTV}_{\min } \mathrm{cGy}(\%)$ & PTV $_{\text {mean }}$ cGy (\%) \\
\hline HN1 & 89.70 & $0.15(0.17)$ & $-0.19(-0.17)$ & 99.58 & $0.48(0.02)$ & $-0.19(0.00)$ \\
\hline HN2 & 82.83 & $-0.03(-0.25)$ & $0.07(0.03)$ & 99.97 & $0.24(0.01)$ & $0.07(0.00)$ \\
\hline HN3 & 84.76 & $-14.38(-14.32)$ & $-0.15(-0.05)$ & 99.93 & $-0.82(-0.02)$ & $-0.15(0.00)$ \\
\hline Thx1 & 83.63 & $1.17(6.32)$ & $0.48(0.19)$ & 99.75 & $2.33(0.03)$ & $0.48(0.00)$ \\
\hline Thx2 & 86.63 & $0.23(0.16)$ & $-0.54(-0.27)$ & 99.72 & $-0.09(0.00)$ & $-0.54(-0.01)$ \\
\hline Thx3 & 87.33 & $-0.08(-0.52)$ & $-0.79(-0.31)$ & 99.79 & $0.39(0.01)$ & $-0.79(-0.01)$ \\
\hline Thx4 & 71.14 & $2.48(1.08)$ & $-0.77(-0.27)$ & 99.89 & $-0.70(-0.01)$ & $-0.77(-0.01)$ \\
\hline Thx5 & 79.44 & $-2.55(-1.14)$ & $-0.56(-0.21)$ & 99.83 & $-2.26(-0.04)$ & $-0.56(-0.01)$ \\
\hline Thx6 & 80.14 & $-0.17(-4.74)$ & $0.35(0.37)$ & 99.99 & $0.83(0.01)$ & $0.35(0.00)$ \\
\hline Thx7 & 81.41 & $-1.58(-1.53)$ & $0.14(0.11)$ & 100 & $-1.70(-0.02)$ & $0.14(0.00)$ \\
\hline Thx8 & 74.98 & $-0.44(-3.18)$ & $-0.13(-0.10)$ & 99.88 & $0.92(0.01)$ & $-0.13(0.00)$ \\
\hline Abd1 & 67.90 & $0.84(0.45)$ & $0.11(0.05)$ & 99.83 & $1.24(0.01)$ & $0.11(0.00)$ \\
\hline Abd2 & 80.76 & $3.07(2.58)$ & $-0.30(-0.16)$ & 98.56 & $-0.10(0.00)$ & $-0.30(0.00)$ \\
\hline Abd3 & 83.19 & $-0.43(-2.73)$ & $-0.46(-0.26)$ & 99.98 & $-0.87(-0.01)$ & $-0.46(-0.01)$ \\
\hline Pel1 & 69.24 & $-0.23(-4.46)$ & $-0.16(-0.27)$ & 100 & $-0.35(0.00)$ & $-0.16(0.00)$ \\
\hline Pel2 & 77.33 & $0.79(0.80)$ & $-0.11(-0.08)$ & 99.90 & $-0.10(0.00)$ & $-0.11(0.00)$ \\
\hline Pel3 & 84.25 & $-0.56(-4.04)$ & $0.29(0.20)$ & 99.99 & $0.92(0.01)$ & $0.29(0.00)$ \\
\hline Leg1 & 88.13 & $0.75(12.66)$ & $-0.32(-0.28)$ & 99.96 & $-0.37(-0.01)$ & $-0.32(0.00)$ \\
\hline Mean & 80.71 & $-0.61(-0.71)$ & $-0.19(-0.08)$ & 99.81 & $-0.00(0.00)$ & $-0.17(-0.00)$ \\
\hline SD & 6.22 & $3.67(5.34)$ & $0.38(0.20)$ & 0.32 & $1.08(0.02)$ & $0.37(0.00)$ \\
\hline
\end{tabular}

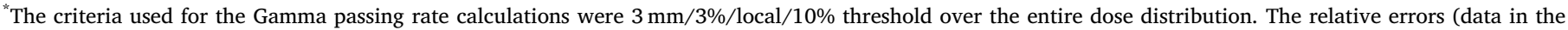
brackets) were normalized to the reference dose as calculated on the treatment isocentre.

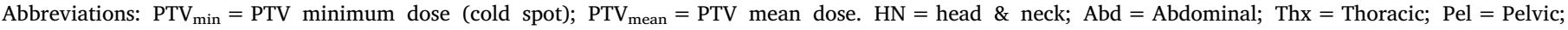
HQ = High Quality; LD = Low dose; SD = standard deviation.

\section{References}

[1] Kim S, Yoshizumi TT, Toncheva G, et al. Comparison of radiation doses between cone beam CT and multi detector CT: TLD measurements. Radiat Prot Dosimetry 2008;132(3):339-45.

[2] Islam MK, Purdie TG, Norrlinger BD, et al. Patient dose from kilovoltage cone beam computed tomography imaging in radiation therapy. Med Phys 2006;33(6):1573-82.

[3] Dzierma, Yvonne, et al. Abdominal imaging dose in radiology and radiotherapy phantom point dose measurements, effective dose and secondary cancer risk. Phys Med 2017;43:49-56.

[4] Zhang $\mathrm{Y}, \mathrm{Wu} \mathrm{H}$, Chen $\mathrm{Z}$, et al. Concomitant imaging dose and cancer risk in imageguided thoracic radiotherapy. Int J Radiat Oncol Biol Phys 2015;93(3):523-31.

[5] Zhang Y, Yan Y, Nath R, et al. Personalized assessment of kV cone beam computed tomography doses in image-guided radiotherapy of pediatric cancer patients. Int $\mathrm{J}$ Radiat Oncol Biol Phys 2012;83(5):1649-54.

[6] Hidalgo Rivas JA, Horner K, Thiruvenkatachari B, et al. Development of a low-dose protocol for cone beam CT examinations of the anterior maxilla in children. Br J Radiol 2015;88(1054):20150559.

[7] Ouyang L, Solberg T, Wang J. Noise reduction in low-dose cone beam CT by incorporating prior volumetric image information. Med Phys 2012;39(5):2569-77.

[8] Alaei P, Ding G, Guan H. Inclusion of the dose from kilovoltage cone beam CT in the radiation therapy treatment plans. Med Phys 2010;37(1):244-8.

[9] Gayou O, Parda DS, Johnson M, et al. Patient dose and image quality from megavoltage cone beam computed tomography imaging. Med Phys 2007;34(2):499-506.

[10] Dzierma, Yvonne, et al. Image quality and dose distributions of three linac-based imaging modalities. Strahlentherapie und Onkologie 2015;191(4):365-74.

[11] Li Y, Netherton T, Nitsch PL, et al. Normal tissue doses from MV image-guided radiation therapy (IGRT) using orthogonal MV and MV-CBCT. J Appl Clin Med Phys 2018;19(3):52-7.

[12] Quinn A, Holloway L, Cutajar D, et al. Megavoltage cone beam CT near surface dose measurements: potential implications for breast radiotherapy. Med Phys 2011;38(11):6222-7. 